\title{
Spondyloepimetaphyseal dysplasia, Isidor type
}

INSERM

\section{Source}

INSERM. (1999). Orphanet: an online rare disease and orphan drug data base.

Spondyloepimetaphyseal dysplasia, Isidor type. ORPHA:370015

Spondyloepimetaphyseal dysplasia, Isidor type is rare primary bone dysplasia disorder characterized by normal birth length with early postnatal growth deficiency resulting in severe disproportionate short stature (with short trunk and limbs), severe genum varum, flexion contractures in the hips and lumbar hyperlordosis. Radiological findings reveal platyspondyly with central indentation of vertebral endplates, progressive and severe epimetaphyseal abnormalities that primarily affect the lower limbs and include very small, irregular proximal femoral and knee epiphyses, severe coxa vara, delayed ossification of proximal femoral epiphyses, and irregular distal femoral and proximal tibial metaphyses. 